\title{
Active Textile Antennas in Professional Garments for Sensing, Localisation and Communication
}

Arnaut Dierck ${ }^{1}$, Sam Agneessens ${ }^{1}$, Frederick Declercq ${ }^{1}$, Bart Spinnewyn ${ }^{1}$, Gert-Jan Stockman $^{1}$, Patrick Van Torre ${ }^{1}$, Luigi Vallozzi ${ }^{1}$, Dries Vande Ginste ${ }^{1}$, Jan Vanfleteren ${ }^{2}$, Thomas Vervust ${ }^{2}$ and Hendrik Rogier ${ }^{1}$

${ }^{1}$ Ghent University-IMEC, Dept. of Information Technology, Sint-Pietersnieuwstraat 41, B-9000 Ghent, Belgium

${ }^{2}$ Ghent University-IMEC, Centre for Microsystems Technology, Technology park 914, B-9052, Gent-Zwijnaarde

New wireless wearable monitoring systems integrated in professional garments require a high degree of reliability and autonomy. Active textile antenna systems may serve as platforms for body-centric sensing, localization and wireless communication systems, in the meanwhile being comfortable and invisible to the wearer. We present a new dedicated comprehensive design paradigm and combine this with adapted signal processing techniques that greatly enhance the robustness and the autonomy of these systems. On the one hand, the large amount of real estate available in professional rescue worker garments may be exploited to deploy multiple textile antennas. On the other hand, the size of each radiator may be designed large enough to ensure high radiation efficiency when deployed on the body. This antenna area is then reused by placing active electronics directly underneath and energy harvesters directly on top of the antenna patch. We illustrate this design paradigm by means of recent textile antenna prototypes integrated in professional garments, providing sensing, positioning and communication capabilities. In particular, a novel wearable active Galileo E1-band antenna is presented and fully characterized, including noise figure and linearity performance.

Keywords:

Corresponding author: Arnaut Dierck; email: arnaut.dierck@intec.ugent.be; phone: +32 9 2643353

\section{Introduction}

In recent years, lots of research was devoted to increase the operational safety and efficiency of police, army and rescue services. In particular, wearable electronic systems greatly enhance the functionality of professional rescue worker garments by providing sensing, localisation and wireless communication capabilities. Smart fabrics and interactive textiles (SFIT) [1,2], which are unobtrusively integrated into garments, do not hinder the movements during interventions, and, in the meanwhile, continuously monitor life signs, activities and environmental conditions, relaying these data wirelessly to a 
remote location for supervision by the operations coordinator. In addition, in hazardous situations, alarms and specific instructions can be fed back to each individual in action. As SFIT systems are to be deployed in harsh conditions and during critical operations, their reliability and autonomy are two key concerns of the designers. To ensure sufficient autonomy without the use of heavy batteries, the electronics must be highly energy-efficient. As a lot of power is consumed in establishing wireless communication links, textile antennas are critical components, so they should preferably exhibit high gain and large radiation efficiency. Garments provide the space needed to deploy antennas with such characteristics, and by making use of a large ground plane, absorption of antenna radiation by the human body is also avoided. Yet, special care must be taken by designers of wearable antennas to counter degradation of antenna performance due to bending, wrinkling and crumpling of the large flexible textile antenna [3-7] as the wearer moves around. Moreover, a good selection of materials is needed to avoid excessive substrate losses due to humidity trapped in the substrate fabric [8] and a thermoplastic polyurethane (TPU) coating may be required to protect the antenna during washing [9]. In addition to increasing the power-efficiency of SFIT systems, energy harvesters may be added to scavenge energy from one or more energy sources available in the neighbourhood of the body, in order to increase the operational autonomy.

In this contribution, we propose a dedicated comprehensive design paradigm to implement energy-efficient active wearable antennas with stable performance when integrated in professional garments that are worn during interventions. To improve reliability and autonomy of the active antenna modules, we integrate electronics on the planar textile antenna's feed plane, directly underneath its ground plane, and position energy harvesters directly on the antenna plane. We outline the measures taken to ensure that the antenna performance is not reduced due to the integration of these additional components. We then pinpoint how this strategy resulted in several implementations for different applications in the context of interventions by rescue workers and public regulatory services, as recently proposed in literature.

In Section II, we present the comprehensive design paradigm to design autonomous active textile antennas based on full-wave/circuit co-design and co-optimization. Integrating active electronic circuits directly underneath the wearable antenna, results in a compact communication module and avoids weak connections that easily break when put under stress during interventions. Moreover, by placing the active electronic circuits directly on the antenna feed plane, the length of radio-frequency (RF) connections is minimized, thereby reducing signal attenuation and improving signal integrity and electromagnetic compatibility of the device. In a next step, energy harvesters can be deployed on the active antennas to increase their autonomy. This is shown by integrating a set of solar cells on top of the antenna patch of a wearable module, without disturbing its radiation characteristics. We then move on to discuss two recent antenna designs for sensing, localisation and wireless communication applications. Section III describes a wearable low-cost through-wall Doppler radar that may be deployed in rescue worker garments to detect moving persons behind walls and victims lying under rubble. In Section IV, we outline the design and validation of a wearable active Galileo E1-band antenna, additionally covering the Galileo Search-and-Rescue (SAR) downlink and Glonass L1 frequencies. Finally, we wrap up by drawing some conclusions in Section V. 


\section{Textile antenna design paradigm}

In order to provide additional functionalities to technical garments for rescue workers, wearable, robust and autonomous modules are required. To this aim, we put forward the dedicated design strategy outlined in Figure 1. Starting from the nominal

application-specific design specifications, the particular operating conditions in which the wearable modules have to operate, dictate the final design requirements, the choice of topology and the material selection. Antenna bending, body presence and environmental parameters, such as heat or moisture, for example, can cause frequency shifts, which may be anticipated by adding safety margins to the frequency range in which the antenna has to comply with the specifications. These adjusted conservative requirements lead to a consequent choice of the active antenna topology. The preliminary circuit and antenna designs are subsequently co-optimized according to the principles described in subsection A). As outlined in subsection B), energy harvesters can then be integrated onto the active antenna, producing a compact, autonomous module that is evaluated in realistic testing conditions.

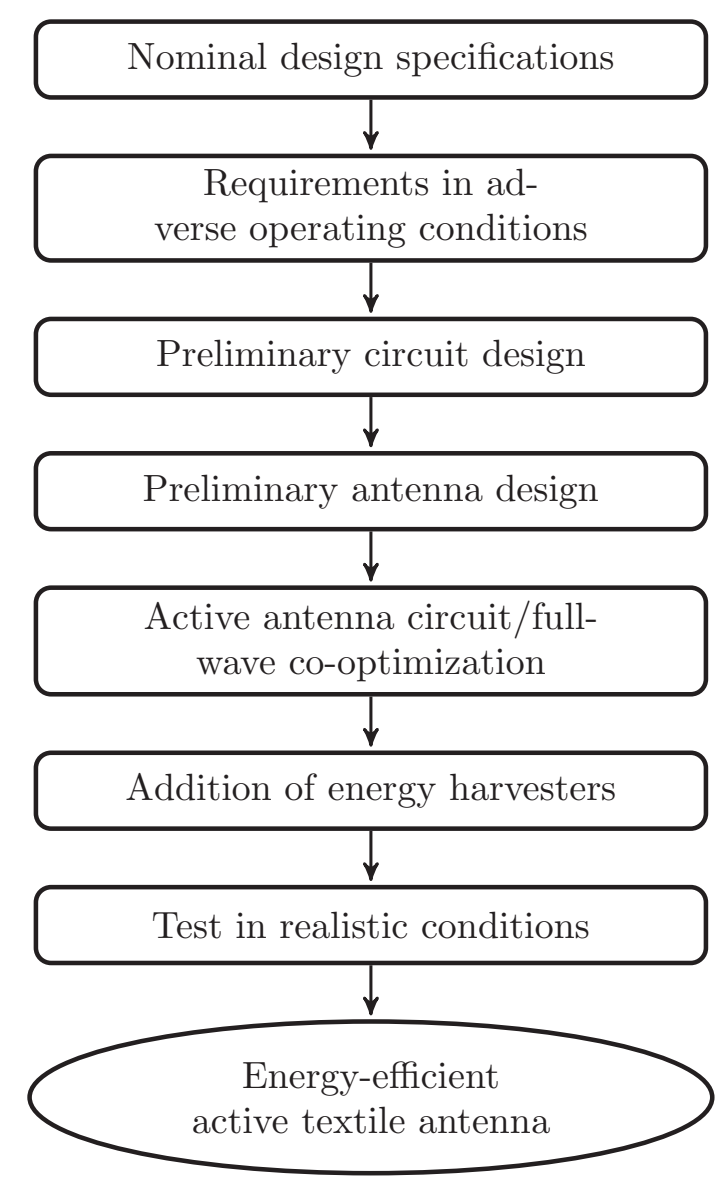

Figure 1: Dedicated energy-efficient active textile antenna design paradigm. 


\section{A) Active antenna circuit/full-wave co-optimization}

Direct integration of active electronics circuits onto the wearable antenna reduces the number of connections and keeps RF paths short. This is, in particular, beneficial for circuits implemented on textile substrates and interconnections with e-textiles, as, on the one hand, substrate and conductive losses are typically more important compared to conventional rigid printed circuit board materials, and, on the other hand, soldering or press-fitting connectors onto conductive textiles results in weak links prone to breaking when subjected to stress incurred, for example, by movements. In addition, via connections should also be avoided, if possible, as these may also come loose when pressure is exerted onto the textile or foam substrate.

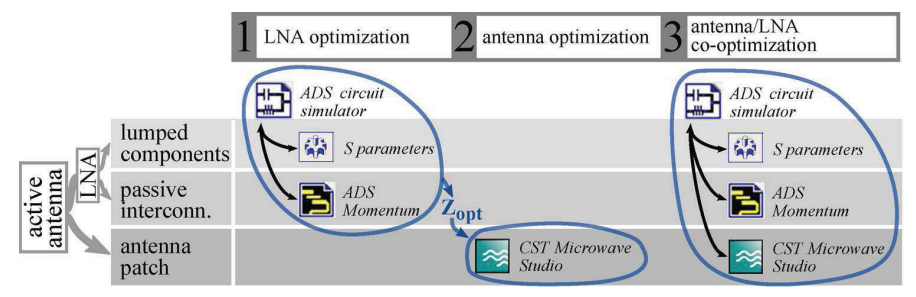

Figure 2: Design strategy for optimal noise characteristics.

The design of active antennas requires a joint circuit/full-wave optimization to simultaneously design the antenna and the active electronics to meet the desired specifications [10-13]. Typically, impedance matching and circular polarization may be desired for the antenna together with large available gain, input and output matching, as well as low noise figure for the low-noise amplifier (LNA) attached to the antenna output. Given the large number of design variables available for optimization, we devised two dedicated strategies to keep the design process manageable [10]. The first process puts forward an optimal complex radiation impedance for the passive textile antenna, resulting in a minimal noise figure at the LNA's output. This impedance is found in the first step of the full-wave/circuit co-optimization procedure for the active electronics only, producing a preliminary optimal LNA design. In the process, a full-wave simulator is applied to generate an N-port scattering matrix describing the interconnections of the active electronics circuit. This scattering matrix is combined with N-port descriptions of the different active and passive lumped components in the circuit in a subsequent circuit simulation. In a second step, full-wave optimization of the passive antenna is performed to fix the antenna dimensions that provide the optimal radiation impedance. In third and final step, the complete active antenna is co-optimized to jointly maximize the performance of both the antenna and the active electronics. This design flow for optimal noise characteristics without the need of a matching network is sketched in Fig. 2. It guarantees short RF connections and avoids excess losses due to components added for matching. The strategy makes use of the most suited simulators for each part of the active textile antenna. In particular, the passive antenna was modelled in the 3D full-wave frequency domain simulator of CST Microwave Studio, which is able to take into account the finite conductivity of the electro-textile Flectron, used as antenna plane and ground plane. As the LNA circuit is implemented on a thin polyimid flex, its interconnections can be modelled by means of the planar-3D full-wave simulator ADS 
Momentum. The reader is referred to $[10,14]$ for further details.

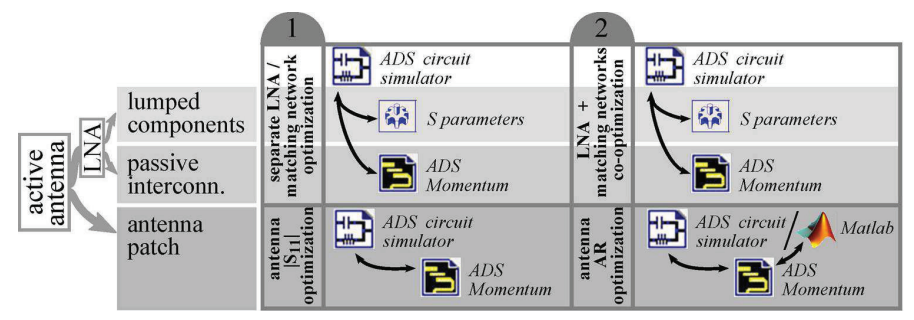

Figure 3: Design strategy for optimal impedance matching.

Antennas for satellite communication typically exhibit stringent requirements in terms of circular polarization. Deforming the radiating structure together with optimization of the feed point may not suffice to meet the requirement that the axial ratio remains below $3 \mathrm{~dB}$ over a sufficiently large bandwidth. If this is the case, the use of a quadrature hybrid should be considered. As implementations in wearable microstrip technology are typically too large at GPS frequencies, miniaturized off-the-shelf lumped hybrids are preferred. Moreover, a filter between the receiving antenna and the LNA may be required to suppress interference and noise. For satellite communication, such filters typically require very steep filter flanks and narrow transition regions between pass band and stop bands. Again, a microstrip implementation of a filter of sufficiently large order will be prohibitively large and an off-the-shelf discrete ceramic, surface acoustic wave (SAW) or Bulk Acoustic Wave (BAW) filter is preferred. As these components have a fixed impedance level of $50 \Omega$ at their ports, the above described co-optimization strategy must be modified. Now, in a first step, to accommodate the discrete component, the antenna output and the LNA input are both separately matched to $50 \Omega$. This requires a matching network to guarantee optimal noise performance for the LNA. In a second step, at the antenna side, axial ratio and antenna impedance are jointly optimized, whereas a full-wave/circuit co-optimization is performed on the complete LNA circuit. This design flow for optimal impedance matching of both the passive antenna and the LNA is sketched in Fig. 3. As all conductive layers are implemented by means of copper patterns on polyimid flex, only the planar-3D full-wave simulator ADS Momentum is used in the design process. More details are found in $[10,15]$.

\section{B) Integration of energy harvesters onto wearable antennas}

An important remaining issue standing in the way of a commercial breakthrough of smart SFIT systems for professional garments concerns ensuring sufficient autonomy without the need of heavy batteries and frequent recharging. Therefore, recent research concentrated on adding energy-scavengers to these systems, in order to collect energy from the body and its environment to power the system [16-19]. For modules in SFIT garments, solar energy and kinetic energy originating from body movement are the most important sources. In [16] it is shown that the antenna patch may serve as a platform for flexible amorphous silicon (a-Si:H) solar cells, thereby reusing the space consumed by the large antenna. In Fig. 4 we show the aperture-coupled shorted wearable solar patch antenna for communication in the $902-928 \mathrm{MHz}$ UHF band. By adopting a PIFA topology for the textile antenna and by routing the feed wires of the flexible solar cells 
along the shorting-wall of the antenna, the antenna radiation is not influenced by the presence of the energy harvester glued onto the antenna patch. Furthermore, only the positive polarity of the solar cell has to be routed through the antenna substrate. The solar cell's cathode can be directly connected to the patch of the antenna since the patch provides DC grounding through the antenna's shorting wall.
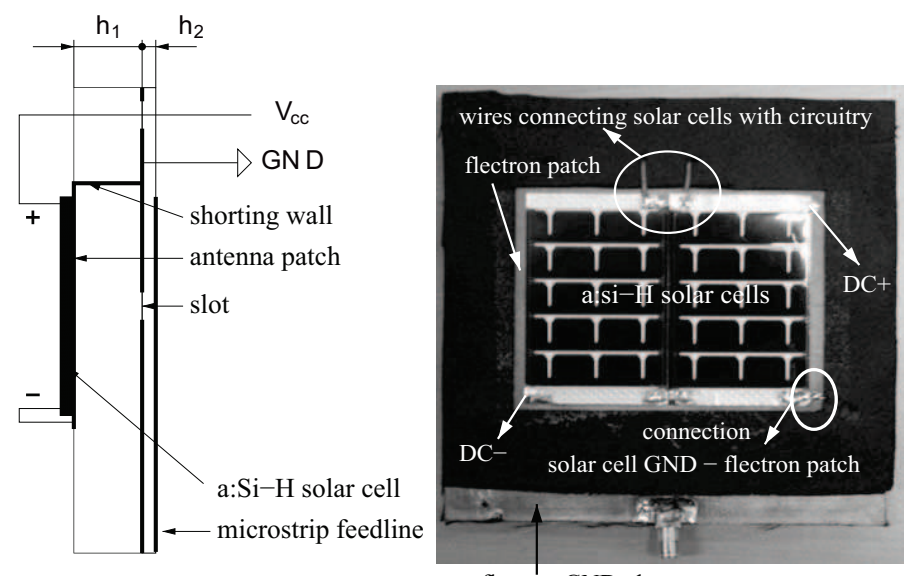

Figure 4: Aperture-coupled shorted wearable solar patch antenna for 902-928 MHz UHF band.

The antenna substrate is a flexible polyurethane foam with a thickness $h_{1}=11 \mathrm{~mm}$, a relative permittivity $\varepsilon_{r}=1.16$ and a loss tangent $\tan \delta=0.010$. The feed substrate is an assembly of two aramid textile layers with a thickness $h_{2}=0.95 \mathrm{~mm}$, an $\varepsilon_{r}=1.97$ and a loss tangent $\tan \delta=0.020$. The conductive patch and ground plane are made out of a copper coated woven nylon fabric, whereas the microstrip feedline is constructed from copper foil. All layers are assembled by means of an adhesive sheet. The antenna design used the time domain solver of CST Microwave Studio in which antenna patch size, microstrip feedline stub length and aperture size were optimized in order to accomplish impedance matching in the $902-928 \mathrm{MHz}$ frequency band. The available antenna surface (= conductive patch) for integration of solar cells is $62 \mathrm{~mm} \times 80 \mathrm{~mm}$, resulting in sufficient space for two solar cells as shown in Fig. 4.

Under ideal illumination conditions, measuring $100 \mathrm{~mW} / \mathrm{cm}^{2}$, the solar cell can deliver up to $57.3 \mathrm{~mW}$ (maximum power point). This illumination level represents sunlight directly overhead on a clear bright day on earth. The current configuration is based on two solar cells and can provide a maximum DC power of about $114 \mathrm{~mW}$. However, in real life applications, the DC output power will be lower since the orientation of the solar cell with respect to the sun and the illumination strength will differ from these optimal conditions. Furthermore, the load connected to the solar cell configuration must be optimal in order to operate in the solar cell's maximum power point.

In order to investigate the influence of the solar cells on the antenna characteristics, both a reflection coefficient and radiation pattern measurement of the antenna with and without solar cells were executed. The simulated reflection coefficient, the measured reflection coefficients of the antenna in free space with and without solar cells, and the measured $\left|S_{11}\right|$ with the antenna positioned on the chest of a human body are presented in Fig. 5. The simulated and measured bandwidth is about $48 \mathrm{MHz}$ whereas the on-body bandwidth increased to $64 \mathrm{MHz}$ due to the additional losses induced by the proximity of 
the human body. Moreover, a slightly larger bandwidth is observed because of the presence of the solar cells which incur a small additional loss. However, this excess loss is much smaller than the extra losses observed when the antenna operates in the vicinity of the human body. The simulated and measured antenna gains in the XZ and YZ-plane are displayed in Fig. 6. Again, a comparison is made between the radiation pattern of the antenna with and without solar cells. The maximum gain of the antenna is about $3 \mathrm{dBi}$, and from these measurements we can conclude that the solar cells have a minor influence on the radiation performance of the antenna.

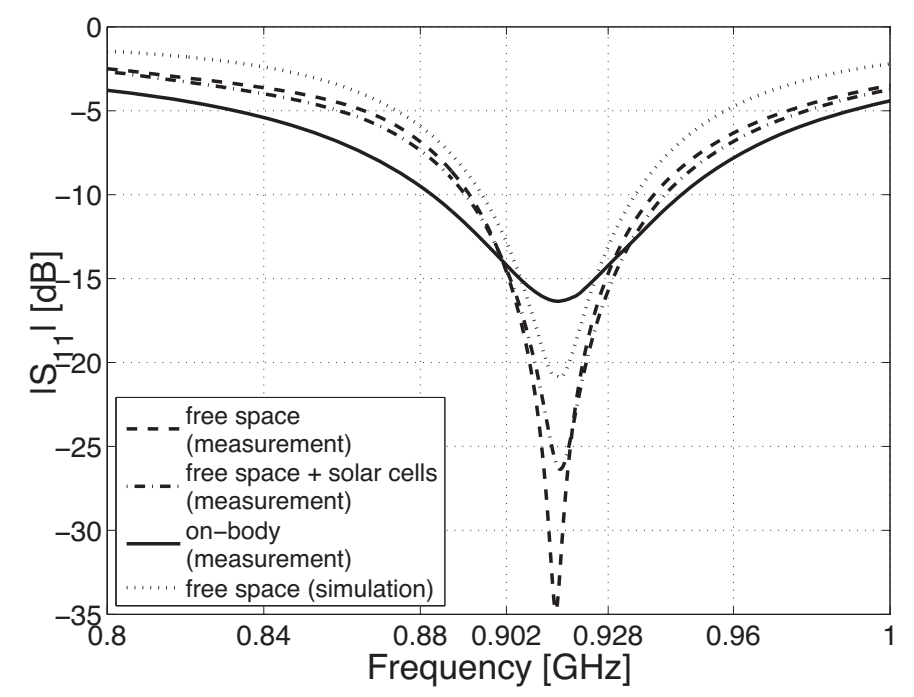

Figure 5: Measured and simulated $\left|S_{11}\right|$ of the aperture coupled shorted wearable solar patch antenna.

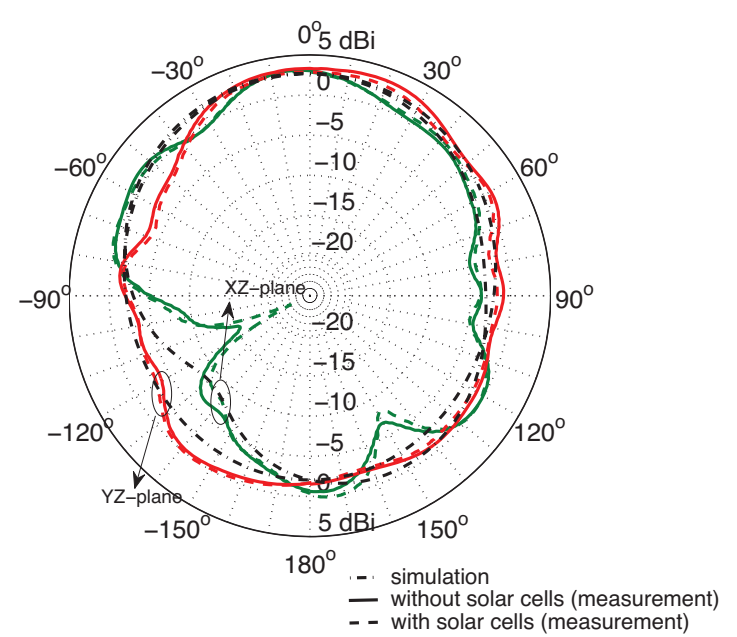

Figure 6: Measured and simulated antenna radiation patterns in the XZ and YZ-plane of the aperture coupled shorted wearable solar patch antenna at $915 \mathrm{MHz}$. 


\section{Wearable through-wall Doppler radar}

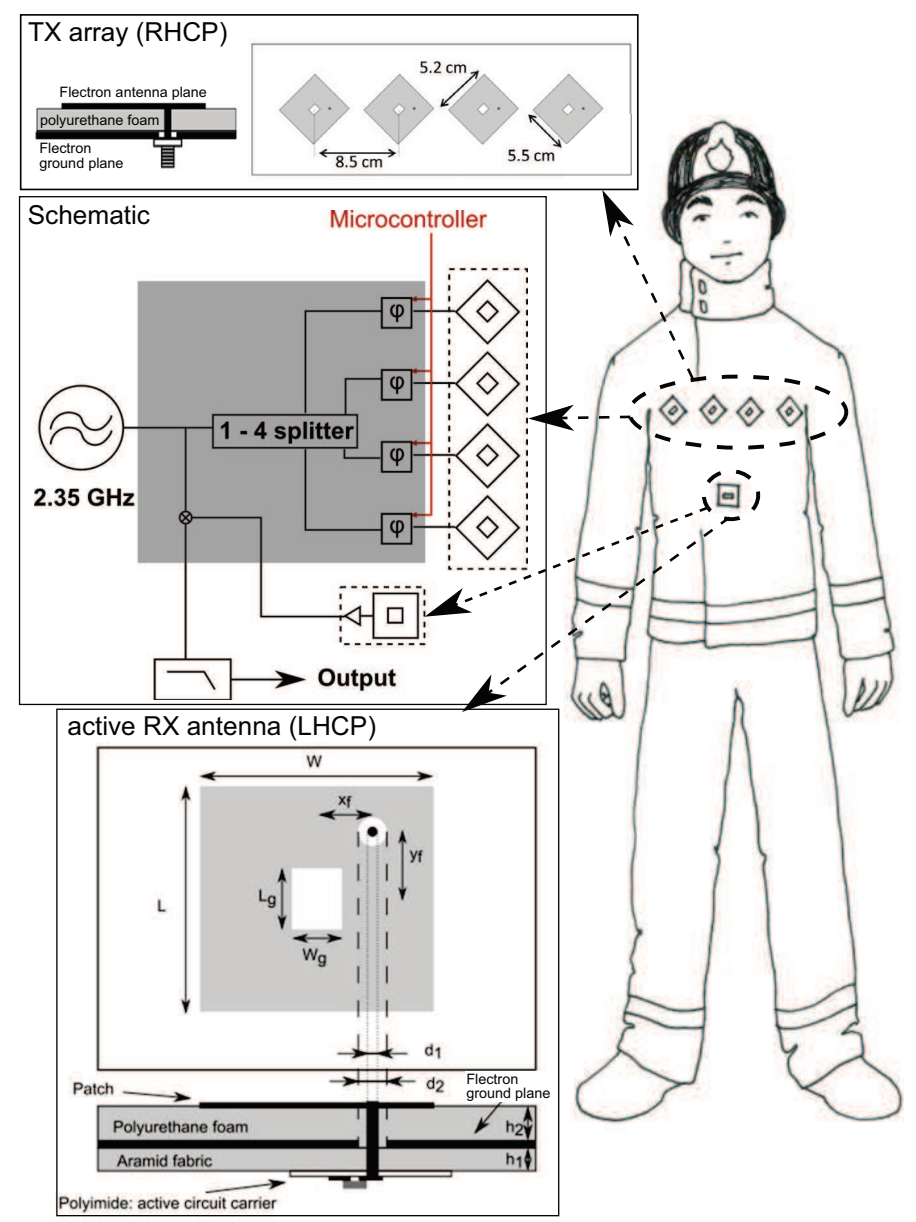

Figure 7: Wearable through-wall Doppler radar (firefighter drawing by Laura Goethals).

The design procedure outlined in Section II was applied to construct a low-cost, low-weight, wearable Doppler radar system capable of detecting moving objects behind a barrier, operating at $2.35 \mathrm{GHz}$. In rescue operations, wearability is critical, since the user's range of motion should not be limited by the radar system. The novelty of this radar system lies in the wearability and its comfortable integration into a garment, which is why a simple, power-efficient Doppler radar architecture was chosen, requiring minimal data processing. As shown in Fig. 7, the transmit part of the Doppler radar consists of a four-element phased array of textile antennas. The beam emitted by this array is right-hand circularly polarized along all scanning angles and provides $9.2 \mathrm{dBi}$ gain. The element spacing of $8.5 \mathrm{~cm}$ results in a large aperture and allows beamsteering from $-15^{\circ}$ to $+15^{\circ}$ with respect to boresight. In figure 8, the $\left|S_{11}\right|$ and $\left|S_{21}\right|$ are depicted. It can be seen that the array is broadly matched in its intended frequency range $(2.3-2.4 \mathrm{GHz})$, ensuring functionality when affected by potential frequency shifts due to antenna bending and body proximity. Moreover, we note that the individual array elements are adequately isolated from each other. In Fig. 9, the radiation pattern of the transmit array is depicted for beams steered in the $-10^{\circ}, 0^{\circ}$ and $15^{\circ}$ direction. Note that, for these steering 


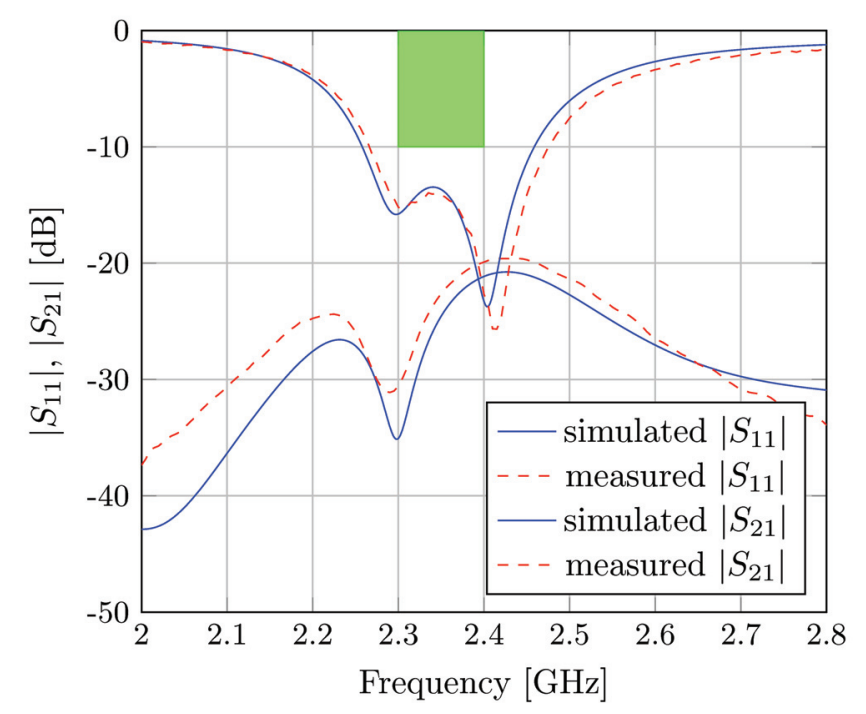

Figure 8: Simulated and measured $\left|S_{11}\right|$ and $\left|S_{21}\right|$ of the wearable four-element array.

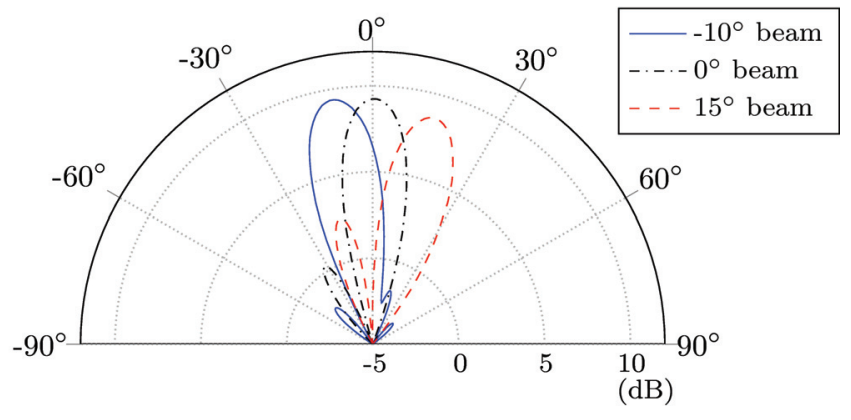

Figure 9: Radiation pattern of the transmit array with beam steered in $-10^{\circ}, 0^{\circ}$ and $15^{\circ}$ direction.

directions, there is a single main beam and grating lobes are absent. At the receiving end, textile fabrics found in professional garments were used to develop an active wearable receive antenna. Applying the design strategy for optimal noise characteristics outlined in Section II and shown in Fig. 2 results in 15.7 dBi gain, $1.1 \mathrm{~dB}$ noise figure, left-hand circular polarization, and a $3 \mathrm{~dB}$ axial ratio beamwidth larger than $50^{\circ}$. The transmit and receive antennas both consist of microstrip patch antennas with a sufficiently large ground plane, providing radiation in a semi-hemisphere away from the body. Therefore, the ground plane effectively shields the antenna from the body or electronics integrated on the antenna backside. In Fig. 10, the measured radiation pattern of the active receive antenna is depicted. The maximum gain is found along the broadside direction and equals $15.7 \mathrm{dBi}$. Fig. 11 shows the spectrogram measured by the radar worn by a rescue worker standing in front of a fire-retardant door, detecting a person walking at $1.5 \mathrm{~m} / \mathrm{s}$ at a position of $15^{\circ}$ with respect to boresight, behind that door. A full description of the design, fabrication and validation of the wearable radar is found in [20]. 


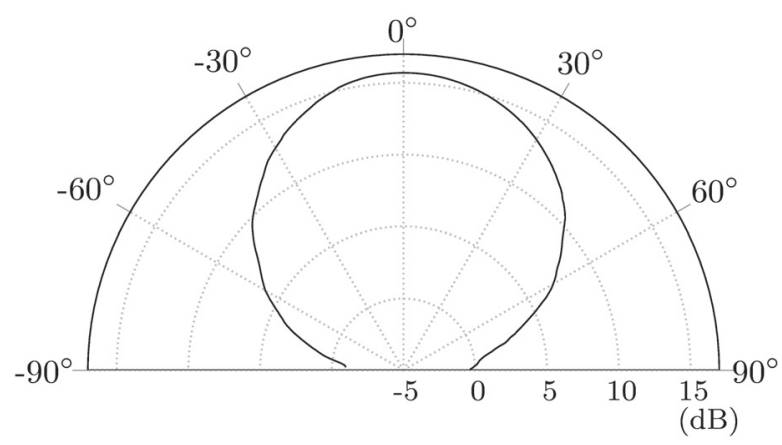

Figure 10: Measured radiation pattern of the active receive antenna.

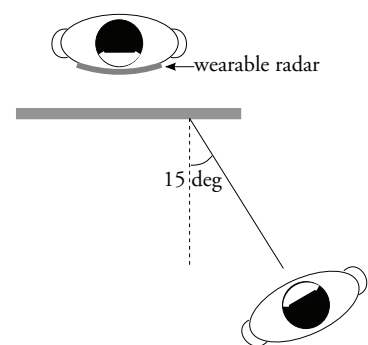

(a)

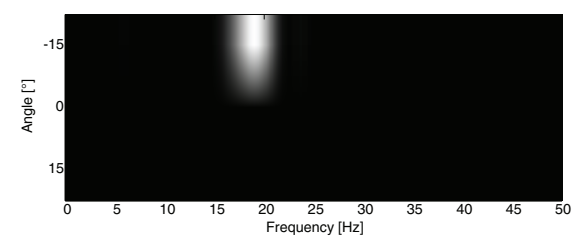

(b)

Figure 11: Measurement setup with a rescue worker wearing the radar, trying to detect a person walking at $15^{\circ}$ behind a closed fire-retardant door (a) and measured spectrogram (b).

\section{Wearable active Galileo E1-band antenna}

The second design strategy outlined in Section II, following Fig. 3, was applied to design an active wearable Galileo E1-band antenna, additionally servicing the Galileo SAR downlink and the Glonass L1 band. In order to provide the aforementioned functionalities, the antenna has to cover a frequency spectrum ranging from $1.544 \mathrm{GHz}$ to $1.611 \mathrm{GHz}$, while being right-handed circularly polarized. Moreover, in terms of application scenario, the antenna is intended for use in rescue-worker garments. This requires a module that is not only compact and flexible, ensuring the wearer's movements are not hindered, but also robust, guaranteeing performance in harsh conditions. In order to achieve these requirements, an aperture-coupled microstrip patch topology [15], as shown in Fig. 12, has been selected. This topology is low-profile and the ground plane shields the antenna from the body, reducing its influence on the radiation performance, and allowing the integration of active electronics on the antenna backside, eliminating potential weak links that would otherwise be present between the antenna and the electronic system. The aperture coupling helps to reduce the amount of vias in the design, increasing robustness to stresses occuring when the antenna is bent or compressed. In order to achieve a wideband circular polarization, a discrete hybrid coupler was preferred over other techniques [21-23]. Moreover, we opted for a compact discrete component instead of a microstrip realization, leaving space for the low-noise amplifier and reducing the vulnerability of the coupler to bending influences. This hybrid coupler is connected to the Maxim MAX2659 LNA [24], providing a high gain and a low 


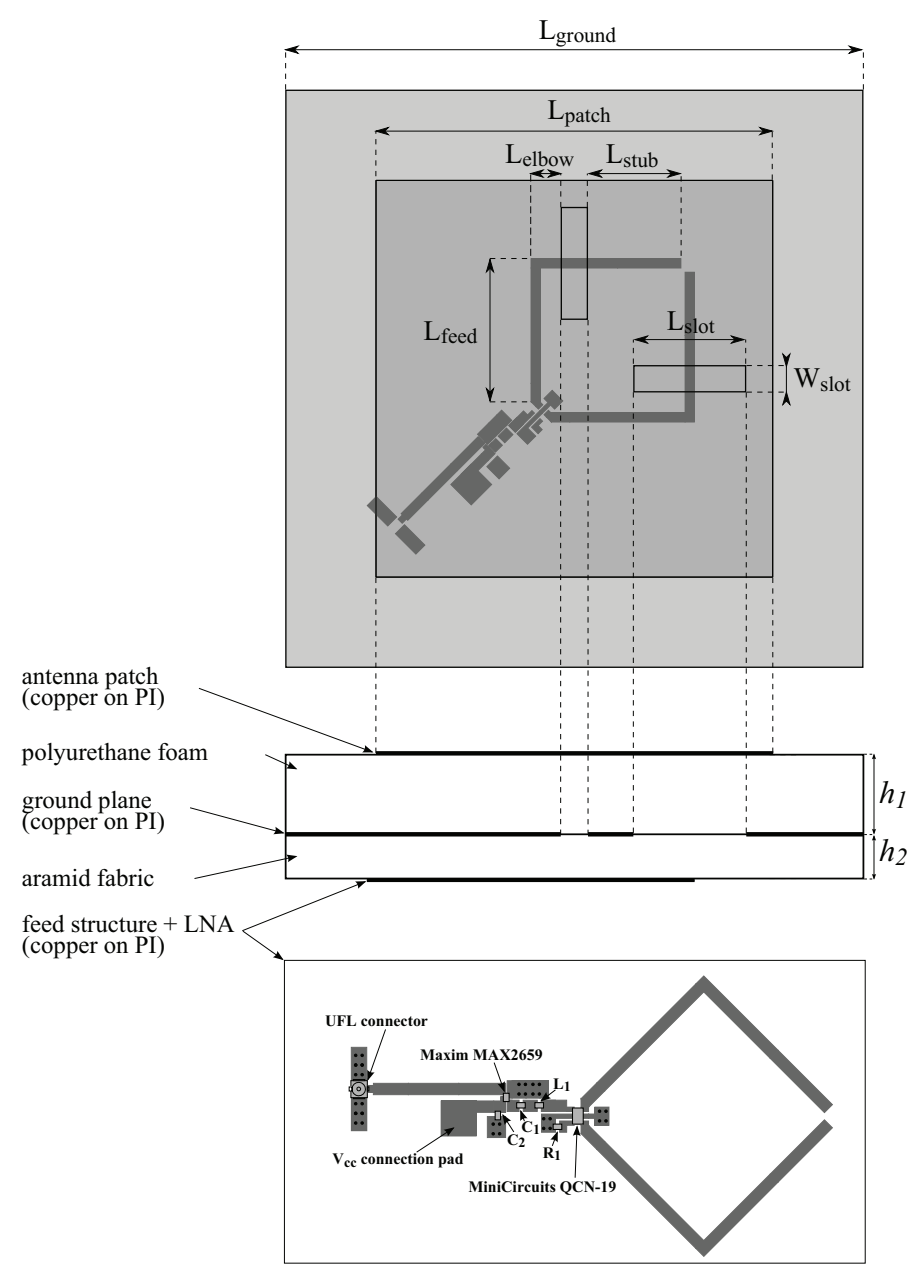

Figure 12: Wearable active Galileo E1-band antenna.

noise figure in a small package that can be conveniently integrated into the feed plane of the antenna. The detailed view in Fig. 12 presents the feed circuit layout, in which the discrete hybrid coupler is connected to the LNA input via an inductor-capacitor matching network $\left(L_{1}=6.8 \mathrm{nH}\right.$ and $\left.C_{1}=470 \mathrm{pF}\right)$. Apart from the discrete coupler, the LNA and the two-element matching network, only two additional components are required (50 $\Omega$-termination $R_{1}$ and $33 \mathrm{nF}$ decoupling capacitor $C_{2}$ ), permitting a small and rugged circuit. As depicted in Fig. 13, measurements indicate that the active antenna is robustly matched over a frequency band ranging from $1.41 \mathrm{GHz}$ to $1.675 \mathrm{GHz}$. The antenna's axial ratio is lower than $3 \mathrm{~dB}$ from $1.465 \mathrm{GHz}$ to $1.765 \mathrm{GHz}$. This wideband circular polarization is achieved by using the discrete hybrid coupler. As depicted in Fig. 14, the antenna gain reaches a maximum of $25.45 \mathrm{~dB}$ at $1.595 \mathrm{GHz}$. It stays within $3 \mathrm{~dB}$ of this maximum value from 1.54 to $1.66 \mathrm{GHz}$.

To characterize the noise and linearity performance of the active antenna, noise measurements were performed on the LNA integrated on an aramid textile substrate, and linearity measurements were performed on the complete active antenna. The noise figure (NF) of the stand-alone LNA at $1.575 \mathrm{GHz}$ is measured to be $0.95 \mathrm{~dB}$, obtained after deembedding the attenuation of the SMA-UFL adapter and the UFL-cable connected to 


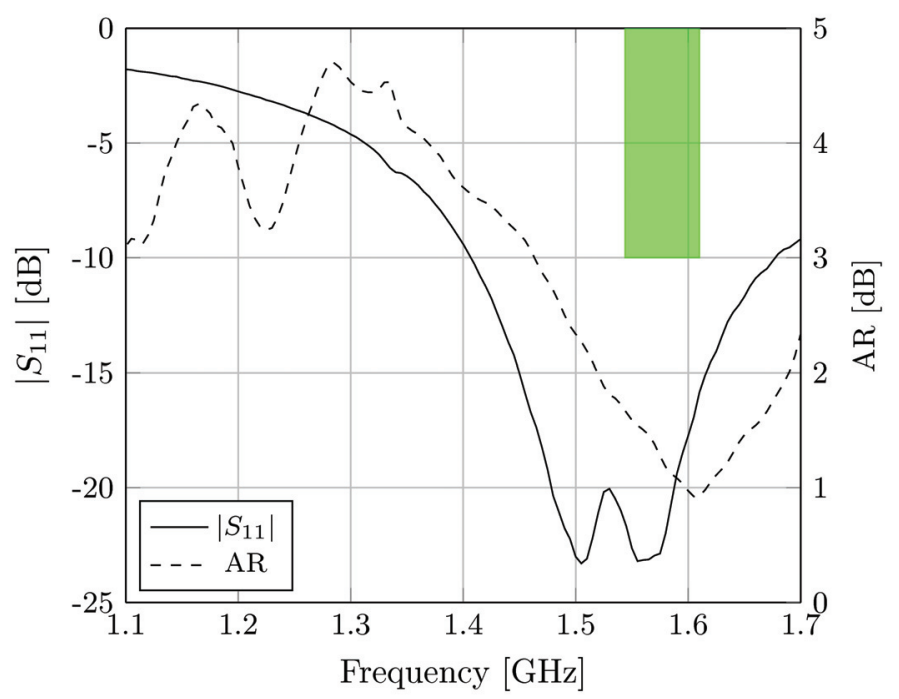

Figure 13: Measured $\left|S_{11}\right|$ and axial ratio of the wearable active Galileo E1-band antenna.

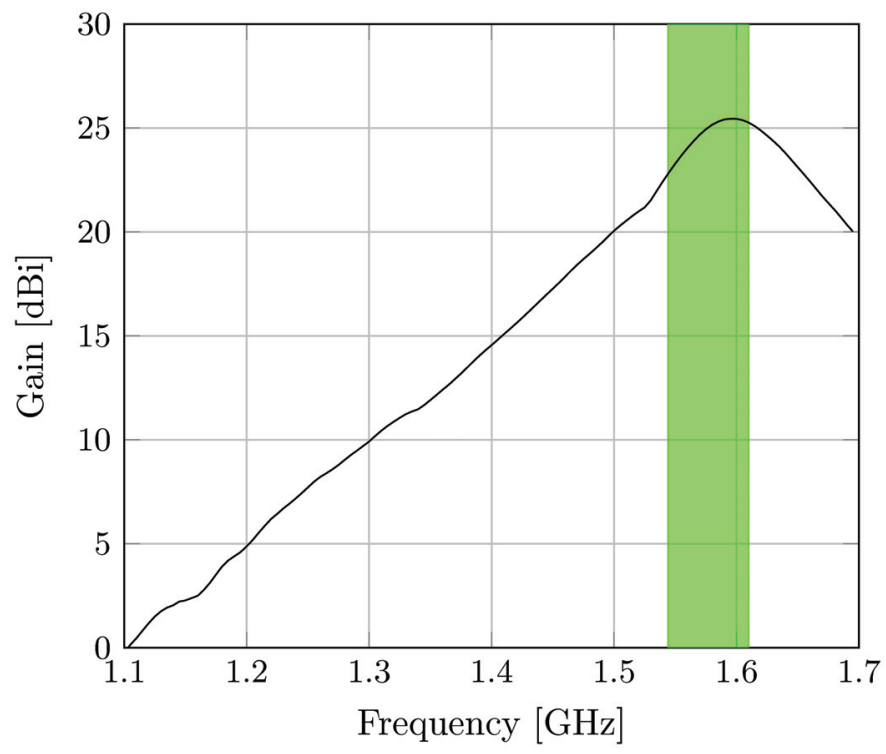

Figure 14: Measured gain of the wearable active Galileo E1-band antenna.

the LNA input. This value is slightly higher than the one listed in the datasheet. The LNA examined here is integrated onto a textile substrate glued to a copper-on-polyimide laminate, on which the circuit is etched. This layered substrate is characterized by a $\tan \delta=0.02$, resulting in a larger attenuation in the circuit's interconnections compared to rigid, high-frequency laminates. However, thanks to the short transmission lines in the LNA circuit, the attenuation and its effect on the noise figure are limited. Yet, the vias in the textile substrate are fabricated by threading copper wires through the substrate, introducing additional inductance in the ground connection of the LNA chip, potentially affecting its performance. Next, by using the Gain Compression and Swept IMD applications available on the Agilent N5242A PNA-X Vector Network Analyzer, which 
use an external power meter to calibrate the PNA-X receivers as reliable power meters, the 1-dB input compression point $(\mathrm{P} 1 \mathrm{~dB})$ and third-order input intercept point (IIP3) of the active antenna were determined. For these measurements, the setup displayed in Fig. 15 was used. Port 1 of the PNA-X is connected to a highly linear low-noise amplifier, which in turn is connected to a standard gain horn. As discussed in [25], the high OIP3 (43 dB from 700 to $1600 \mathrm{MHz}$ ) of the MiniCircuits ZRL-3500+ amplifier allows it to be driven with an input power level up to $0 \mathrm{dBm}$ before intermodulation distortion is generated, providing ample range for the linearity characterization of the active antenna under test. At a distance of $3.55 \mathrm{~m}$ from the horn, the active antenna, displayed on the figure as a seperate antenna and LNA block, is aligned with the horn. The active antenna is fed by two AA batteries, resulting in a $3.15 \mathrm{~V}$ feed voltage. The active antenna is then connected to port 2 of the PNA-X. In Fig. 15, detailed descriptions and gains/losses of the different elements of the setup are specified. In this setup, the PNA-X is located in a Faraday cage, while the other components were positioned in an anechoic chamber. To be able to compare the results with the values listed in the MAX2659 datasheet, the same $5 \mathrm{MHz}$ tone spacing and $-40 \mathrm{dBm} /$ tone power (at the LNA input) were used for the IIP3 measurement. The values for the P1dB and IIP3 returned by the PNA-X measurements were corrected with the term $G_{L N A 1}+G_{H o r n}+L+G_{\text {ant }}=-4.9 \mathrm{~dB}$ to take into account the gains/losses that occur before the signal transmitted at port 1 of the PNA-X actually reaches the LNA integrated into the active antenna. In this way, a P1dB of $-9.965 \mathrm{dBm}$ and a IIP3 of $-4.23 \mathrm{dBm}$ at $1.575 \mathrm{GHz}$ were measured. These results are in in good agreement with the values indicated in the datasheet.

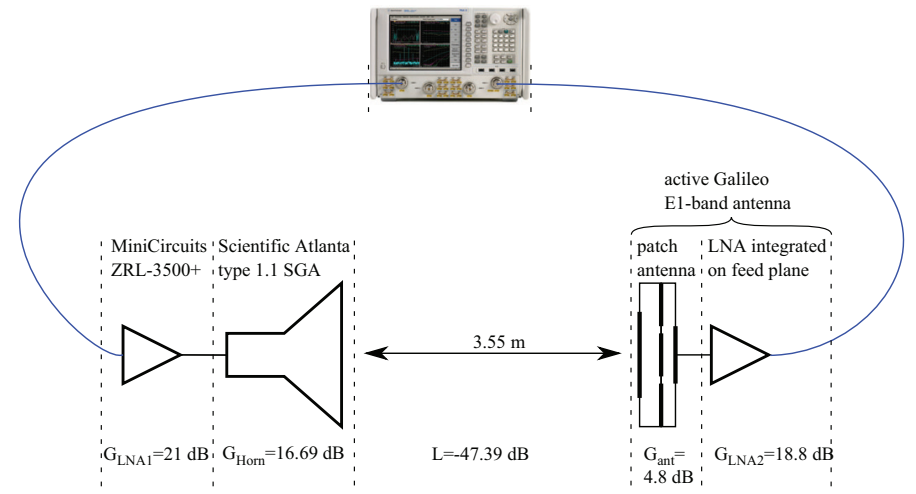

Figure 15: Setup for the active antenna P1dB and IIP3 measurements.

\section{Conclusion}

Smart electronic systems for sensing, localization and wireless communications should exhibit robustness, reliability and a high degree of autonomy while not adding too much weight, nor hindering the movements of the wearer. In this paper, we exploit the large area available in professional garments to integrate flexible textile antennas. In turn, the wearable antenna is used as a platform for the integration of active electronics, on the feed plane directly below the ground plane, as well as for the integration of solar cells directly on top of the antenna patch. We presented a comprehensive dedicated design 
paradigm that yields optimal active antenna characteristics. The two outlined full-wave/circuit co-optimization strategies were applied to the design of a wearable through-wall Doppler radar for the detection of moving persons behind barriers and of a wearable active Galileo E1-band antenna, whose noise and linearity performance were characterized. The large area available in garments can also be exploited to deploy multiple antennas to improve the signal quality by means of diversity and MIMO techniques. For more details, we refer to [26-29].

\section{ACKNOWLEDGEMENTS}

This work was co-funded by the European Commission in the context of the "Antenna and fRontend MOdules for pUblic Regulated Service applications (ARMOURS)", EC-FP7 Galileo.2011.3.12: Collaborative Project, Grant agreement no.: 287166, by BELSPO through the IAP Phase VII BESTCOM project and by the Fund for Scientific Research Flanders (FWO-V).

\section{REFERENCES}

[1] D. Curone, E. Secco, L. Caldani, A. Lanata, R. Paradiso, A. Tognetti, and G. Magenes, "Assessment of sensing fire fighters uniforms for physiological parameter measurement in harsh environment," Information Technology in Biomedicine, IEEE Transactions on, vol. 16, no. 3, pp. 501-511, 2012.

[2] T. Kennedy, P. Fink, A. Chu, N. Champagne, G. Lin, and M. Khayat, "Body-worn E-Textile antennas: The good, the low-mass, and the conformal," IEEE Trans. Antennas Propag., vol. 57, no. 4, pp. 910-918, 2009.

[3] A. Tronquo, H. Rogier, C. Hertleer, and L. Van Langenhove, "Robust planar textile antenna for wireless body LANs operating in $2.45 \mathrm{GHz}$ ISM band," IEE Electronic Letters, vol. 42, no. 3, pp. 142-146, 2006.

[4] I. Locher, M. Klemm, T. Kirstein, and G. Troster, "Design and characterization of purely textile patch antennas," IEEE Transactions on Advanced Packaging, vol. 29, pp. 777-788, December 2006.

[5] P. Salonen and Y. Rahmat-Samii, "Textile antennas: Effects of antenna bending on input matching and impedance bandwidth," IEEE Aerospace and Electronic Systems Magazine, vol. 22, pp. 18-22, December 2007.

[6] C. Hertleer, H. Rogier, L. Vallozzi, and L. Van Langenhove, "A textile antenna for off-body communication integrated into protective clothing for firefighters," IEEE Trans. on Antennas and Propagation, vol. 57, pp. 919-925, April 2009.

[7] J. Lilja, P. Salonen, T. Kaija, and P. de Maagt, "Design and manufacturing of robust textile antennas for harsh environments," Antennas and Propagation, IEEE Transactions on, vol. 60, no. 9, pp. 4130-4140, 2012.

[8] C. Hertleer, A. Van Laere, H. Rogier, and L. Van Langenhove, "Influence of Relative Humidity on Textile Antenna Performance," Textile Research Journal, vol. 80, pp. 177-183, JAN 2010 . 
[9] M. L. Scarpello, I. Kazani, C. Hertleer, H. Rogier, and D. Vande Ginste, "Stability and Efficiency of Screen-Printed Wearable and Washable Antennas," IEEE Antennas and Wireless Propagation Letters, vol. 11, pp. 838-841, 2012.

[10] A. Dierck, F. Declercq, and H. Rogier, "Review of active textile antenna co-design and optimization strategies," in RFID-Technologies and Applications (RFID-TA), 2011 IEEE International Conference on, pp. 194 -201, sept. 2011.

[11] M. Del Prete, D. Masotti, N. Arbizzani, and A. Costanzo, "Remotely identify and detect by a compact reader with mono-pulse scanning capabilities," Microwave Theory and Techniques, IEEE Transactions on, vol. 61, no. 1, pp. 641-650, 2013.

[12] D. Masotti, P. Francia, A. Costanzo, and V. Rizzoli, "Rigorous electromagnetic/circuit-level analysis of time-modulated linear arrays," Antennas and Propagation, IEEE Transactions on, vol. 61, no. 11, pp. 5465-5474, 2013.

[13] D. Masotti, A. Costanzo, M. Prete, and V. Rizzoli, "Genetic-based design of a tetra-band high-efficiency radio-frequency energy harvesting system," Microwaves, Antennas Propagation, IET, vol. 7, no. 15, pp. 1254-1263, 2013.

[14] F. Declercq and H. Rogier, "Active Integrated Wearable Textile Antenna With Optimized Noise Characteristics," IEEE Trans. on Antennas and Propagation, vol. 58, pp. 3050-3054, Sep. 2010.

[15] A. Dierck, H. Rogier, and F. Declercq, "A wearable active antenna for global positioning system and satellite phone," Antennas and Propagation, IEEE Transactions on, vol. 61, pp. 532-538, Feb. 2013.

[16] F. Declercq, A. Georgiadis, and H. Rogier, "Wearable Aperture-Coupled Shorted Solar-Patch Antenna for Remote Tracking and Monitoring Applications," in Fifth European Conference on Antennas and Propagation - EuCAP 2011, (Rome, Italy), pp. 2992-2996, Apr. 2011.

[17] A. Collado and A. Georgiadis, "Conformal hybrid solar and electromagnetic (em) energy harvesting rectenna," Circuits and Systems I: Regular Papers, IEEE Transactions on, vol. 60, no. 8, pp. 2225-2234, 2013.

[18] S. V. Shynu, M. Roo Ons, M. Ammann, S. McCormack, and B. Norton, "Dual band a-si:h solar-slot antenna for 2.4/5.2ghz wlan applications," in Antennas and Propagation, 2009. EuCAP 2009. 3rd European Conference on, pp. 408-410, 2009.

[19] M. B. Schubert and J. H. Werner, "Flexible solar cells for clothing," Materials Today, vol. 9, no. 6 , pp. $42-50,2006$.

[20] S. Agneessens, P. Van Torre, F. Declercq, B. Spinnewyn, G.-J. Stockman, H. Rogier, and D. Vande Ginste, "Design of a Wearable, Low-Cost, Through-Wall Doppler Radar System," International Journal of Antennas and Propagation, no. 840924, 2012.

[21] E. Kaivanto, M. Berg, E. Salonen, and P. de Maagt, "Wearable circularly polarized antenna for personal satellite communication and navigation," IEEE Transactions on Antennas and Propagation, vol. 59, pp. $4490-4496$, dec. 2011. 
[22] L. Vallozzi, W. Vandendriessche, H. Rogier, C. Hertleer, and M. L. Scarpello, "Wearable textile GPS antenna for integration in protective garments," in Proc. of the Fourth European Conference on Antennas and Propagation (EuCAP), 2010. 4th European Conference on Antennas and Propagation (EuCAP), Barcelona, SPAIN, APR 12-16, 2010.

[23] P. Salonen, Y. Rahmat-Samii, M. Schaffrath, and M. Kivikoski, "Effect of textile materials on wearable antenna performance: a case study of GPS antennas," in 2004 IEEE Antennas and Propagation Society Int. Symp., vol. 1, pp. 459 - 462 Vol.1, june 2004.

[24] Maxim Integrated Products, GPS/GNSS Low Noise Amplifier, MAX2659 data sheet, August 2011.

[25] A. Biondi, F. Declercq, D. De Zutter, H. Rogier, and L. Vallozzi, "Electromagnetic compatibility aware design and testing of intermodulation distortion under multiple co-located sources illumination," IET Science Measurement \&5 Technology, vol. 6, pp. 105-112, MAR 2012 .

[26] L. Vallozzi, P. Van Torre, C. Hertleer, H. Rogier, M. Moeneclaey, and J. Verhaevert, "Wireless Communication for Firefighters Using Dual-Polarized Textile Antennas Integrated in Their Garment," IEEE Trans. Antennas Propag., vol. 58, pp. 1357-1368, APR 2010.

[27] P. Van Torre, L. Vallozzi, C. Hertleer, H. Rogier, M. Moeneclaey, and J. Verhaevert, "Indoor Off-Body Wireless MIMO Communication With Dual Polarized Textile Antennas," IEEE Trans. on Antennas and Propagation, vol. 59, pp. 631-642, Feb. 2011.

[28] P. Van Torre, M. L. Scarpello, L. Vallozzi, H. Rogier, M. Moeneclaey, D. Vande Ginste, and J. Verhaevert, "Indoor Off-Body Wireless Communication: Static Beamforming versus Space-Time Coding," Intern. Journal of Antennas and Propagation, 2012.

[29] P. Van Torre, L. Vallozzi, L. Jacobs, H. Rogier, M. Moeneclaey, and J. Verhaevert, "Characterization of Measured Indoor Off-Body MIMO Channels with Correlated Fading, Correlated Shadowing and Constant Path Loss," IEEE Trans. on Wireless Communications, vol. 11, pp. 712-721, Feb. 2012.

\section{Bibliographies}

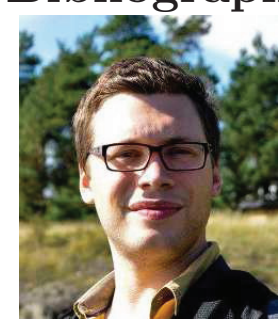

Arnaut Dierck was born in 1987. He received the M. Sc. Degree in electrical engineering from Ghent University, Ghent, Belgium in 2010. Since September 2010 he has been working as a Ph.D. student at the electromagnetics group in the Department of Information Technology (INTEC) at Ghent University. His research activities concentrate on the design of active textile antennas for wearable applications. 
Sam Agneessens was born in 1986. In 2011 received the M. Sc. Degree in electrical engineering from Ghent University, Ghent, Belgium. He is currently working towards his Ph.D. at the Department of Information Technology (INTEC) at Ghent University. His research interests are textile antennas and SIW techniques for wearable systems and Radar.

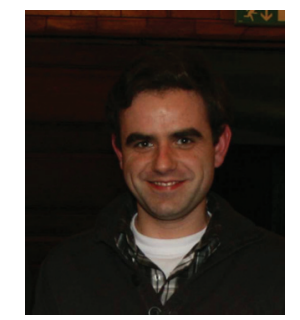

Frederick Declercq was born in 1983. He received the Master of Science degree in electronic engineering at Howest, university college West Flanders, Kortrijk, Belgium. He received the Ph.D. degree in electrical engineering at the faculty of engineering at Ghent University in 2011.

Since September 2005, he has been with the electromagnetics group, department of Information Technology (INTEC) at Ghent University. He is currently conducting his postdoctoral research at IMEC, Ghent University. His research interests are electromagnetic characterization of textile materials and flexible foam materials, the design of wearable active textile antennas and energy scavenging techniques for wireless sensor nodes applied in body area networks.

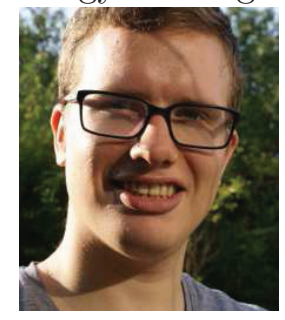

Bart Spinnewyn was born in 1989. He received the M. Sc. Degree in electrical engineering with greatest distinction from Ghent University, Ghent, Belgium in 2012. Since September 2012 he has been working as an analog designer at Caeleste CVBA. His research activities concentrate on CMOS image sensor design.

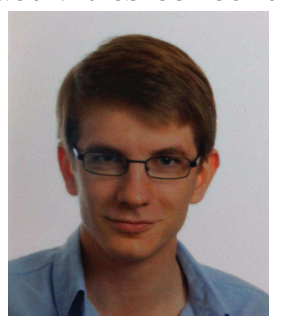

Gert-Jan Stockman was born in 1989. He receiver the M. Sc. Degree in electrical engineering from Ghent University, Ghent, Belgium in 2012 with greatest distinction. Since September 2012 he has been working as a Ph.D. student at the electromagnetics group in the Department of Information Technology (INTEC) at Ghent University. His research activities concentrate on computational electromagnetics and more specifically domain decomposition techniques. 


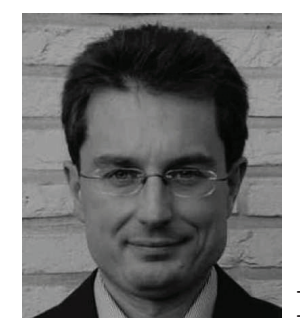

Patrick Van Torre received the Electrical Engineering degree (ing.) in 1995 and pursued the Ph.D. degree at Ghent University, Gent, Belgium from 2008 till 2012. He is employed by Ghent University, at the Faculty of Applied Engineering Sciences since 1999, where he teaches theory courses in Electronics and ICT, organizes project-oriented lab sessions and is involved in public relations activities as well as hardware development projects for third parties. He is a part-time postdoctoral researcher at the Department of Information Technology at Ghent University, active in the field of wireless communication, focusing on body-centric multiple-input multiple-output (MIMO) and beam-forming systems.

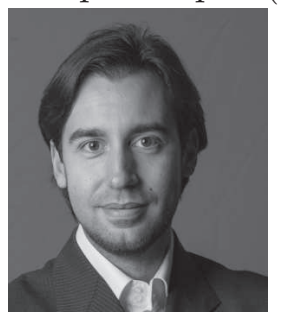

Luigi Vallozzi was born in Ortona, Italy, in 1980. He received the Laurea degree in electronic engineering from the Universit Politecnica delle Marche, Ancona, Italy, in 2005 and the Ph.D. degree in electrical engineering at Ghent University, Ghent, Belgium, in 2010. He is currently working as a Postdoctoral Research Fellow of the Fund for Scientific Research Flanders (FWO) within the Electromagnetics Group at the Information Technology Department (INTEC) of Ghent University. His research focuses on design and prototyping of antennas for wearable textile systems, and the modeling and characterization of multiple-input multiple-output wireless communication systems.

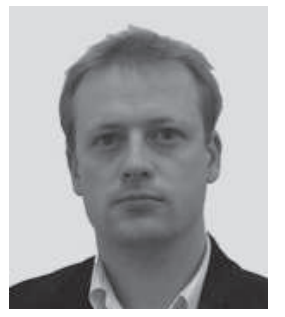

Dries Vande Ginste was born in 1977. He received the M.S. degree and the Ph.D. degree in electrical engineering from Ghent University, Gent, Belgium, in 2000 and 2005, respectively. He is currently an Associate Professor with the Electromagnetics Group, Department of Information Technology, Ghent University. In June and July 2004, he was a Visiting Scientist at the Department of Electrical and Computer Engineering, University of Illinois at Urbana-Champaign (UIUC), IL, USA. From September to November 2011, he was a Visiting Professor at the EMC Group, Dipartimento di Elettronica, Politecnico di Torino, Italy. His research interests comprise computational electromagnetics, electromagnetic compatibility, signal and power integrity, and antenna design. Dr. Vande Ginste was awarded the International Union of Radio Science (URSI) Young Scientist Award at the 2011 URSI General Assembly and Scientific Symposium, the Best Poster Paper Award at the 2012 IEEE Electrical Design of Advanced Packaging and Systems Symposium (EDAPS) and the Best Paper Award at the 2013 IEEE Workshop on Signal and Power Integrity (SPI). He is a Senior Member of the IEEE. 
Jan Vanfleteren received the Ph.D. degree in electronic engineering from Ghent University, Ghent, Belgium, in 1987. He is currently a Senior Engineer with the Center for Microsystems Technology Group (CMST), Ghent University-Interuniversity Microelectronics Center, and is involved in the development of novel interconnection, assembly, and polymer microsystem technologies, especially for wearable and implantable electronics, biomedical, microfluidics, cell culturing, and tissue engineering applications. He is a Project Manager at CMST, where he has a long-standing experience in coordination and cooperation in EC-funded projects. In 2004, he became a part-time Professor with Ghent University. He is the co-author of over 200 papers in international journals and conferences. He holds 14 patents and patent applications. He is a member of IEEE, IMAPS and MRS.

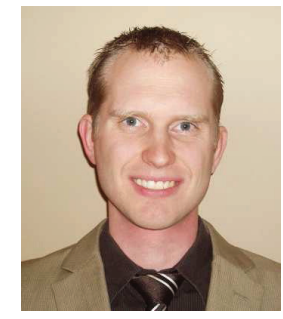

Thomas Vervust is a research scientist at the Center for Microsystems Technology (CMST) at Ghent University in Belgium (affiliated with IMEC). His research focuses on the development of stretchable electronic technologies for large area applications and smart textiles. He received his master degree in Electronics Engineering from Ghent University in 2007 and a $\mathrm{PhD}$ on stretchable and washable electronics for embedding in textiles at Ghent University in 2012.

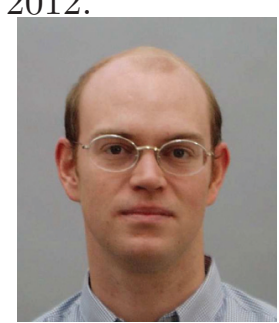

Hendrik Rogier was born in 1971. He received the Electrical Engineering and the Ph.D. degrees from Ghent University, Gent, Belgium, in 1994 and in 1999, respectively. He is a currently a Full Professor with the Department of Information Technology of Ghent University, Belgium, Guest Professor at IMEC, Heverlee, Belgium, and Visiting Professor at the University of Buckingham, UK. From October 2003 to April 2004, he was a Visiting Scientist at the Mobile Communications Group of Vienna University of Technology. He authored and coauthored about 90 papers in international journals and about 110 contributions in conference proceedings. He is serving as a member of the Editorial Board of IET Science, Measurement Technology and acts as the URSI Commission B representative for Belgium. Within the IEEE Microwave Theory and Techniques Society, he is a member of Technical Committee 24 on RFID technology and within the European Microwave Association, he is a member of the Governing Board of Topical Group MAGEO on Microwaves in Agriculture, Environment and Earth Observation. His current research interests are antenna systems, radiowave propagation, body-centric communication, numerical electromagnetics, electromagnetic compatibility and power/signal integrity. 
Dr. Rogier was twice awarded the URSI Young Scientist Award, at the 2001 URSI Symposium on Electromagnetic Theory and at the 2002 URSI General Assembly. In addition, he received the Best Poster Paper Award at the 2012 IEEE Electrical Design of Advanced Packaging and Systems Symposium (EDAPS), the Best Paper Award at the 2013 IEEE Workshop on Signal and Power Integrity (SPI) and the Joseph Morrissey Memorial Award for the First best scientific paper at BioEM 2013. He is a Senior Member of the IEEE. 